\title{
Dialysis Catheter Obstruction
}

National Cancer Institute

\section{Source}

National Cancer Institute. Dialysis Catheter Obstruction. NCI Thesaurus. Code C114764.

Blockage of a dialysis catheter by a thrombus or other substance. 